Article

\title{
Identification of Yeasts with Mass Spectrometry during Wine Production
}

\author{
Miroslava Kačániová ${ }^{1,2, *} \mathbb{0}$, Simona Kunová ${ }^{3}$, Jozef Sabo ${ }^{1}$, Eva Ivanišová ${ }^{4}$, Jana Žiarovská ${ }^{5}$, \\ Soňa Felsöciová ${ }^{6}\left[\right.$ and Margarita Terentjeva ${ }^{7}$ (1)
}

1 Department of Fruit Sciences, Viticulture and Enology, Faculty of Horticulture and Landscape Engineering, Slovak University of Agriculture, Tr. A. Hlinku 2,94976 Nitra, Slovakia; sabododik@gmail.com

2 Department of Bioenergy, Food Technology and Microbiology, Institute of Food Technology and Nutrition, University of Rzeszow, Zelwerowicza St. 4, 35601 Rzeszow, Poland

3 Department of Food Hygiene and Safety, Faculty of Biotechnology and Food Sciences, Slovak University of Agriculture, Tr. A. Hlinku 2, 94976 Nitra, Slovakia; simona.kunova@uniag.sk or simona.kunova@gmail.com

4 Department of Technology and Quality of Plant Products, Faculty of Biotechnology and Food Sciences, Slovak University of Agriculture, Tr. A. Hlinku 2, 94976 Nitra, Slovakia; eva.ivanisova@uniag.sk

5 Department of Plant Genetics and Breeding, Faculty of Agrobiology and Food Resources, Slovak University of Agriculture, Tr. A. Hlinku 2, 94976 Nitra, Slovakia; jana.ziarovska@uniag.sk

6 Department of Microbiology, Faculty of Biotechnology and Food Sciences, Slovak University of Agriculture, Tr. A. Hlinku 2, 94976 Nitra, Slovakia; sona.felsociova@uniag.sk

7 Institute of Food and Environmental Hygiene, Faculty of Veterinary Medicine, Latvia University of Life Sciences and Technologies, K. Helmana iela 8, LV-3004 Jelgava, Latvia; margarita.terentjeva@llu.lv

* Correspondence: miroslava.kacaniova@gmail.com; Tel.: +421-905-499-166

Received: 16 November 2019; Accepted: 3 January 2020; Published: 7 January 2020

\begin{abstract}
The aim of the present study was to identify yeasts in grape, new wine "federweisser" and unfiltered wine samples. A total amount of 30 grapes, 30 new wine samples and 30 wine samples (15 white and 15 red) were collected from August until September, 2018, from a local Slovak winemaker, including Green Veltliner (3), Mūller Thurgau (3), Palava (3), Rhein Riesling (3), Sauvignon Blanc (3), Alibernet (3), André (3), Blue Frankish (3), Cabernet Sauvignon (3), and Dornfelder (3) grapes; federweisser and unfiltered wine samples were also used in our study. Wort agar (WA), yeast extract peptone dextrose agar (YPDA), malt extract agar (MEA) and Sabouraud dextrose agar (SDA) were used for microbiological testing of yeasts. MALDI-TOF Mass Spectrometry (Microflex LT/SH) (Bruker Daltonics, Germany) was used for the identification of yeasts. A total of 1668 isolates were identified with mass spectrometry. The most isolated species from the grapes was Hanseniaspora uvarum, and from federweisser and the wine-Saccharomyces cerevisiae.
\end{abstract}

Keywords: yeasts; grape; federweisser; wine; microbiota identification; MALDI-TOF MS Biotyper

\section{Introduction}

Yeasts naturally occur in wines and vineyards and are especially common on the grapes. Population of yeast species on the grape is not constant and increases during the ripening process. Kloeckera apiculata is a lemon-like cell shape yeast, which colonizes the grape surface [1]. Kloeckera apiculata comprises more than $50 \%$ of the total healthy grape microbiota. Other yeasts like Kloeckera were isolated from the surface of the grapes, which included mainly genera Metschnikowia, Candida, Cryptococcus, Pichia, Rhodotorula, Zygosaccharomyces or Kluyveromyces [2]. The presence of yeasts of the genus Aureobasidium attracted attention as a transitional genus between yeast and microscopic fungi. All the yeasts associated with natural microbiota of grapes are wild yeast strains or non-saccharomyces. Despite the presence of those yeasts on the surface of grapes, the wine production consists of subsequent fermentation stages, 
which are typical for only particular yeast genera [3]. The Saccharomyces genus is the most important for the wine making process; however, this yeast is found on the grapes only in very small amounts. Previous studies that counted Saccharomyces on grapes found as little as $50 \mathrm{CFU} / \mathrm{g}$. Mostly wild yeasts cultures could be found on the grapes and in freshly pressed must with colonization rates of $10^{3}$ to $10^{5} \mathrm{CFU} / \mathrm{mL}$. During alcoholic fermentation, Saccharomyces cerevisiae is dominant, while yeasts in the Pichia and Candida genera are widespread in finished wine. The osmotolerant yeasts Zygosaccharomyces were reported in wines with higher content of residual sugar; yeasts of the Brettanomyces genus were common for wines in barrels $[4,5]$.

The most important yeasts associated with wine production were Hanseniaspora uvarum (anamorph Kloeckera apiculata), Metschnikowia pulcherrima, Rhodotorula mucilaginosa, Rhodotorula glutinis, Aureobasidium pullulans, Cryptococcus magnus, Pichia manshurica, Pichia membranifaciens (anamorph Candida valida), Pichia fermentans, Pichia kluyveri, Pichia occidentalis (anamorph Candida sorbosa), Wickerhamomyces anomalus (anamorph Candida pelliculosa; Pichia anomala is synonymous), Cyberlindnera jadinii (Pichia jadinii is synonymous), Kregervanrija fluxuum (anamorph Candida vini), Candida stellata, Candida inconspicua, Meyerozyma guilliermondii, Zygosaccharomyces bailii, Brettanomyces bruxellensis (teleomorph Dekkera bruxellensis), Saccharomycodes ludwigii, Torulaspora delbrueckii and Saccharomyces cerevisiae. Kluyveromyces marxianus and Debaryomyces hansenii associated with grapes and are known as a contaminant in wine production. The microbiota of grapes creates better conditions for the growth of yeasts rather than bacteria. Low $\mathrm{pH}(\mathrm{pH} 3-3.3)$, high content of sugars (mainly glucose) in grapes, and an anaerobic environment in must are necessary for ethanol fermentation of sugars, converting them into alcohol (ethanol) and $\mathrm{CO}_{2}$ [5-8].

The aim of this study was to identify yeasts in grapes, federweisser and wine samples.

\section{Materials and Methods}

\subsection{Collection of Grape, Federweisser and Wine Samples}

An amount of 90 samples, including grape berries $(n=30)$, federweisser $(n=30)$ and wine $(n=30)$ of Vitis vinifera were collected aseptically in the viticultural area of Vrbové (approximately $48^{\circ} 37^{\prime} 12^{\prime \prime} \mathrm{N}$ and $017^{\circ} 43^{\prime} 25^{\prime \prime} \mathrm{E}$ ) in 2018 . The grape berry samples were transported on ice and stored at $-20^{\circ} \mathrm{C}$ until processing. The white grape varieties Green Veltliner, Mūller Thurgau, Palava, Rhein Riesling and Sauvignon Blanc as well as red grape varieties Alibernet, André, Blue Frankish, Cabernet Sauvignon and Dornfelder were collected. Three sampling points in distal spatial points of different rows were used for sampling of grape berries. Grape samples were collected in August, and processed independently.

Samples of "federweisser" were collected at the end of August 2018 and in the middle of September 2018 from the same winery as the grapes. Samples were collected into $200 \mathrm{~mL}$ sterile plastic bottles and stored at $8 \pm 1{ }^{\circ} \mathrm{C}$ in a refrigerator. Before testing, the samples $(n=30)$ were diluted with sterile physiological saline $(0.85 \%)$. A total of $100 \mu \mathrm{L}$ of each dilution $\left(10^{-1}\right.$ to $\left.10^{-5}\right)$ was used for microbiological testing.

An amount of $200 \mathrm{~mL}$ of each unfiltered wine (before microfiltration) and immediately after were stored at $6-8{ }^{\circ} \mathrm{C}$ in a refrigerator. Collected wine samples were fermented with Saccharomyces cerevisiae in the producing process. The samples were later incubated in the laboratory at room temperature $\left(25 \pm 2{ }^{\circ} \mathrm{C}\right)$ for one week until the laboratory testing was initiated.

\subsection{Cultivation Media}

Wort agar (WA) (HiMedia, Mumbai, India), yeast extract peptone dextrose agar (YPDA) (Conda, Madrid, Spain), malt extract agar (MEA) (Biomark, Maharashtra, India) and Sabouraud dextrose agar (SDA) (Conda, Madrid, Spain) were used for identification of yeasts. All media were supplemented with chloramphenicol (100 mg/L) to inhibit bacterial growth. Chloramphenicol (Biolife, Monza, Italy) was added into cultivation media before sterilization by autoclaving at $115-121^{\circ} \mathrm{C}$ for $15 \mathrm{~min}$. The acid base indicator bromocresol green (BG, Biolofe, Monza, Italy) $(20 \mathrm{mg} / \mathrm{L})(\mathrm{pH}$ range: $3.8-5.4)$ was 
added into the MEA and WA cultivation media before sterilization. Media for yeast cultivation were inoculated with $100 \mu \mathrm{L}$ of the sample suspension. Inoculated agars were incubated at $25{ }^{\circ} \mathrm{C}$ for 3-5 days and the yeasts were identified by colony morphology (colour, surface, edge and elevation) and reinoculated onto trypton soya agar (TSA) (Oxoid, Basingstoke, UK). Yeast species were identified with a MALDI-TOF MS Biotyper.

\subsection{Identification of Isolates with Mass Spectrometry}

Qualitative analysis of yeasts isolates was performed with MALDI-TOF mass spectrometry (Bruker Daltonics, Bremen, Germany). Isolates were put in $300 \mu \mathrm{L}$ of distilled water and $900 \mu \mathrm{L}$ of ethanol, and the suspension centrifuged for $2 \mathrm{~min}$ at 14,000 rpm. The pellet was centrifuged repeatedly and allowed to dry. An amount of $30 \mu \mathrm{L}$ of $70 \%$ formic acid was added to the pellet and $30 \mu \mathrm{L}$ of acetonitrile. Tubes were centrifuged for $2 \mathrm{~min}$ at $14,000 \mathrm{rpm}$ and $1 \mu \mathrm{L}$ of the supernatant was used for MALDI identification. Once dry, every spot was overlaid with $1 \mu \mathrm{L}$ of an HCCA matrix and left to dry at room temperature before analysis. Generated spectra were analyzed on a MALDI-TOF Microflex LT (Bruker Daltonics, Bremen, Germany) instrument using Flex Control 3.4 software and Biotyper Realtime Classification 3.1 with BC-specific software. Criteria for reliable identification were a score of $\geq 2.0$ at species level [9].

\subsection{Statistical Analysis}

The statistical processing of the data obtained from each evaluation was done with Statgraphics Plus version 5.1 (AV Trading, Umex, Dresden, Germany). For each replication the mean was calculated, and the data set were log transformed. Descriptive statistics and logical-cognitive methods and one-way analysis ANOVA were used in the evaluation and statistical analysis.

\section{Results and Discussion}

Grapes are inhabited by versatile microbial groups and have a complex microbial ecology, including filamentous fungi, yeasts and bacteria. These microorganisms pose different physiological characteristics and may affect the wine quality. Some species of parasitic fungi or environmental bacteria might be only found in grapes, while other microorganisms like yeast, lactic acid and acetic acid bacteria occur during the winemaking process [10].

The yeast count in grape ranged from 2.34 (Greener Veltliner) to 2.67 (Dornfelder) log CFU/g on MEA, from 2.19 (Mūller Thurgau) to 2.38 (Dornfelder) log CFU/g on WA, from 2.46 (Greener Veltliner) to 2.66 (Dornfelder) $\log$ CFU/g on YPDA, and from 1.55 (Greener Veltliner) to 1.88 (Dornfelder) log CFU/g on SDA. The colonization of grapes with yeasts is shown in Table 1.

Table 1. Yeasts counts in grape berries on different media.

\begin{tabular}{ccccc}
\hline \multirow{2}{*}{ Sample } & MEA & WA & YPDA & SDA \\
\cline { 2 - 5 } & \multicolumn{4}{c}{ Microbial Counts log CFU/g } \\
\hline Green Veltliner & $2.37 \pm 0.14$ & $2.22 \pm 0.06$ & $2.46 \pm 0.05$ & $1.55 \pm 0.14$ \\
Mūller Thurgau & $2.34 \pm 0.09$ & $2.19 \pm 0.04$ & $2.49 \pm 0.04$ & $1.66 \pm 0.22$ \\
Palava & $2.36 \pm 0.13$ & $2.20 \pm 0.07$ & $2.52 \pm 0.01$ & $1.69 \pm 0.17$ \\
Rhein Riesling & $2.43 \pm 0.01$ & $2.17 \pm 0.06$ & $2.51 \pm 0.02$ & $1.67 \pm 0.16$ \\
Sauvignon Blanc & $2.40 \pm 0.03$ & $2.19 \pm 0.04$ & $2.49 \pm 0.02$ & $1.65 \pm 0.12$ \\
Alibernet & $2.64 \pm 0.10$ & $2.26 \pm 0.08$ & $2.53 \pm 0.03$ & $1.73 \pm 0.16$ \\
André & $2.66 \pm 0.07$ & $2.33 \pm 0.10$ & $2.57 \pm 0.06$ & $1.79 \pm 0.05$ \\
Blue Frankish & $2.64 \pm 0.03$ & $2.36 \pm 0.06$ & $2.59 \pm 0.06$ & $1.82 \pm 0.04$ \\
Cabernet Sauvignon & $2.66 \pm 0.03$ & $2.34 \pm 0.09$ & $2.64 \pm 0.01$ & $1.84 \pm 0.02$ \\
Dornfelder & $2.67 \pm 0.05$ & $2.38 \pm 0.04$ & $2.66 \pm 0.04$ & $1.88 \pm 0.06$ \\
\hline
\end{tabular}

WA-wort agar; YPDA-yeast extract peptone dextrose agar; MEA-malt extract agar; SDA-Sabouraud dextrose agar. 
ANOVA analysis was performed to inspect the significant differences among the microbial count for individual wine varieties when different cultivation media were used (Table 2).

Table 2. One-way ANOVA for analyzed wine varieties-grapes.

\begin{tabular}{ccccccc}
\hline $\begin{array}{c}\text { Cultivation } \\
\text { Media }\end{array}$ & Source & $\begin{array}{c}\text { Sum of } \\
\text { Squares }\end{array}$ & $\begin{array}{c}\text { Degrees of } \\
\text { Freedom }\end{array}$ & $\begin{array}{c}\text { Mean } \\
\text { Square }\end{array}$ & F Statistic & $p$-Value \\
\hline \multirow{4}{*}{ MEA } & treatment & 0.5779 & 9 & 0.0642 & 9.55 & $1.65 \times 10^{-5}$ \\
& error & 0.1347 & 20 & 0.0067 & & \\
& total & 0.7125 & 29 & & & \\
\hline \multirow{2}{*}{ WA } & treatment & 0.1755 & 9 & 0.0195 & 4.26 & 0.0025 \\
& error & 0.0868 & 20 & 0.0043 & & \\
& total & 0.2623 & 29 & & & \\
YPDA & treatment & 0.1179 & 9 & 0.0131 & 8.74 & $3.77 \times 10^{-5}$ \\
& error & 0.0307 & 20 & 0.0015 & & \\
& total & 0.1487 & 29 & & & 0.1287 \\
\multirow{2}{*}{ SDA } & treatment & 0.2863 & 9 & 0.0318 & 1.13 & \\
& error & 0.3513 & 20 & 0.0176 & & \\
\hline
\end{tabular}

WA—wort agar; YPDA—yeast extract peptone dextrose agar; MEA—malt extract agar; SDA—Sabouraud dextrose agar.

Statistically significant differences among microbial counts for individual cultivation media were found in three of the four cultivation media used (Table 3).

Table 3. Significant differences among analyzed grape varieties for individual cultivation media.

\begin{tabular}{|c|c|c|}
\hline Treatments Pair & Tukey HSD $p$-Value & Tukey HSD Inferfence \\
\hline \multicolumn{3}{|l|}{ MEA } \\
\hline A vs. F & 0.0180304 & $* p<0.05$ \\
\hline A vs. G & 0.0085086 & $* * * 0.01$ \\
\hline A vs. $\mathrm{H}$ & 0.0180304 & $* p<0.05$ \\
\hline A vs. I & 0.0076352 & $* * p<0.01$ \\
\hline A vs. J & 0.0068497 & $* * p<0.01$ \\
\hline B vs. F & 0.0085086 & $* * p<0.01$ \\
\hline B vs. G & 0.0039776 & $* * p<0.01$ \\
\hline B vs. $\mathrm{H}$ & 0.0085086 & $* * p<0.01$ \\
\hline B vs. I & 0.0035659 & $* * p<0.01$ \\
\hline B vs. J & 0.0032002 & $* * p<0.01$ \\
\hline C vs. F & 0.0130929 & $* p<0.05$ \\
\hline C vs. G & 0.0061450 & $* * p<0.01$ \\
\hline C vs. H & 0.0130929 & $* p<0.05$ \\
\hline C vs. I & 0.0055127 & $* * p<0.01$ \\
\hline C vs. J & 0.0049454 & $* * p<0.01$ \\
\hline E vs. G & 0.0247440 & $* p<0.05$ \\
\hline E vs. I & 0.0222763 & $* p<0.05$ \\
\hline E vs. J & 0.0200452 & $* p<0.05$ \\
\hline \multicolumn{3}{|l|}{ WA } \\
\hline D vs. H & 0.0395384 & $* p<0.05$ \\
\hline D vs. J & 0.0206542 & $* p<0.05$ \\
\hline \multicolumn{3}{|l|}{ YPDA } \\
\hline A vs. $\mathrm{H}$ & 0.0262122 & $* p<0.05$ \\
\hline A vs. I & 0.0010053 & $* * p<0.01$ \\
\hline A vs. J & 0.0010053 & $* * p<0.01$ \\
\hline B vs. I & 0.0068426 & $* * p<0.01$ \\
\hline
\end{tabular}


Table 3. Cont.

\begin{tabular}{ccc}
\hline Treatments Pair & Tukey HSD $p$-Value & Tukey HSD Inferfence \\
\hline YPDA & & \\
\hline B vs. J & 0.0011070 & $* * p<0.01$ \\
C vs. J & 0.0085840 & $* * p<0.01$ \\
D vs. I & 0.0210350 & $* p<0.05$ \\
D vs. J & 0.0034566 & $* * p<0.01$ \\
E vs. I & 0.0043409 & $* * p<0.01$ \\
E vs. J & 0.0010053 & $* p<0.01$ \\
F vs. J & 0.0168484 & $* 0.05$
\end{tabular}

A-Green Veltliner; B-Mūller Thurgau; C-Palava; D-Rhein Riesling; E-Sauvignon Blanc; F-Alibernet; G-André; H-Blue Frankish; I-Cabernet Sauvignon; J-Dornfelder; WA-wort agar; YPDA-yeast extract peptone dextrose agar; MEA—malt extract agar; SDA—Sabouraud dextrose agar.

Different studies have evaluated the surface microbiota of grape berries due to a possible impact on the hygienic state of the grapes and the direct influence on the winemaking process and wine quality [11-18].

The yeasts count in "federweisser" ranged from 3.51 in Greener Veltliner and Palava to $3.80 \log \mathrm{CFU} / \mathrm{mL}$ in Dornfelder on MEA. On WA, the yeasts count from 3.30 in Palava to $3.53 \log \mathrm{CFU} / \mathrm{mL}$ in Dornfelder were observed. On YPDA, the yeasts count varied from 3.24 in Rhein Riesling to $3.45 \log$ CFU/mL in Dornfelder, and from 3.13 (Sauvignon Blanc) to 3.33 (Dornfelder) $\log \mathrm{CFU} / \mathrm{mL}$ on SDA. Yeasts counts in federweisser are summarized in Table 4.

Table 4. Yeast counts in "federweisser" on different media.

\begin{tabular}{ccccc}
\hline \multirow{2}{*}{ Sample } & MEA & WA & YPDA & SDA \\
\cline { 2 - 5 } & \multicolumn{4}{c}{$\log$ CFU/g } \\
\hline Green Veltliner & $3.51 \pm 0.15$ & $3.41 \pm 0.06$ & $3.37 \pm 0.14$ & $3.21 \pm 0.01$ \\
Mūller Thurgau & $3.54 \pm 0.10$ & $3.38 \pm 0.06$ & $3.30 \pm 0.04$ & $3.19 \pm 0.03$ \\
Palava & $3.51 \pm 0.05$ & $3.30 \pm 0.06$ & $3.27 \pm 0.04$ & $3.16 \pm 0.05$ \\
Rhein Riesling & $3.58 \pm 0.06$ & $3.34 \pm 0.01$ & $3.24 \pm 0.01$ & $3.14 \pm 0.02$ \\
Sauvignon Blanc & $3.56 \pm 0.10$ & $3.36 \pm 0.05$ & $3.27 \pm 0.05$ & $3.13 \pm 0.02$ \\
Alibernet & $3.67 \pm 0.08$ & $3.40 \pm 0.06$ & $3.29 \pm 0.06$ & $3.17 \pm 0.03$ \\
André & $3.70 \pm 0.07$ & $3.43 \pm 0.02$ & $3.36 \pm 0.05$ & $3.18 \pm 0.06$ \\
Blue Frankish & $3.74 \pm 0.02$ & $3.46 \pm 0.05$ & $3.39 \pm 0.05$ & $3.24 \pm 0.09$ \\
Cabernet Sauvignon & $3.76 \pm 0.05$ & $3.48 \pm 0.06$ & $3.41 \pm 0.01$ & $3.30 \pm 0.04$ \\
Dornfelder & $3.80 \pm 0.07$ & $3.53 \pm 0.03$ & $3.45 \pm 0.06$ & $3.33 \pm 0.01$ \\
\hline
\end{tabular}

WA-wort agar; YPDA—yeast extract peptone dextrose agar; MEA—malt extract agar; SDA—Sabouraud dextrose agar.

In study in Slovakia [19], the highest yeasts counts were on MEA for Pinot Noir-6.43 log CFU/mL and the lowest for Moravian Muscat $-4.62 \log \mathrm{CFU} / \mathrm{mL}$. The highest yeasts count on WA were in Pinot Noir-6.39 $\log$ CFU/mL, but the lowest in Irsai Oliver-5.38 $\log$ CFU $/ \mathrm{mL}$. The highest count of yeasts on wild yeast medium (WYM) was in Blue Frankish $6.33 \log$ CFU/mL and the lowest in Dornfelder $4.20 \log \mathrm{CFU} / \mathrm{mL}[19]$.

As the results show, a higher number of yeasts were detected in "federweisser" than in grape. The young wine is a product of fermentation where S. cerevisiae was mostly found. Other species like Hanseniaspora uvarum, Metschnikowia pulcherrima or the genera Pichia or Candida may be present during the individual fermentation stages when the alcohol content do not exceed $4-6 \%[5,20]$. The main microbiota of the grape is the yeast Hanseniaspora uvarum followed by Metschnikowia pulcherrima [4]. These species also initiate the pre-alcoholic fermentation but are being replaced by the dominant $S$. cerevisiae 3-4 days after fermentation. Saccharomyces cerevisiae starts to multiply within 20 days after inoculation into the must [21]. 
ANOVA analysis was performed to inspect the significant differences among the microbial count for individual wine varieties when different cultivation media were used (Table 5).

Table 5. One-way ANOVA results for the analyzed wine varieties-federweisser.

\begin{tabular}{ccccccc}
\hline $\begin{array}{c}\text { Cultivation } \\
\text { Media }\end{array}$ & Source & $\begin{array}{c}\text { Sum of } \\
\text { Squares }\end{array}$ & $\begin{array}{c}\text { Degrees of } \\
\text { Freedom }\end{array}$ & $\begin{array}{c}\text { Mean } \\
\text { Square }\end{array}$ & F Statistic & $p$-Value \\
\hline \multirow{4}{*}{ MEA } & treatment & 0.3717 & 9 & 0.0413 & 6.36 & 0.0002 \\
& error & 0.1234 & 20 & 0.0062 & & \\
& total & 0.4951 & 29 & & & \\
\hline \multirow{3}{*}{ WA } & treatment & 0.1305 & 9 & 0.0145 & 5.23 & 0.0007 \\
& error & 0.0525 & 20 & 0.0026 & & \\
& total & 0.1831 & 29 & & & \\
YPDA & treatment & 0.1426 & 9 & 0.0158 & 3.36 & \\
& error & 0.0818 & 20 & 0.0041 & & \\
& total & 0.2244 & 29 & & & \\
\hline \multirow{2}{*}{ SDA } & treatment & 0.1194 & 9 & 0.0133 & 6.91 & \\
& error & 0.0397 & 20 & 0.0020 & & \\
& total & 0.1591 & 29 & & & \\
\hline
\end{tabular}

WA—wort agar; YPDA—yeast extract peptone dextrose agar; MEA—malt extract agar; SDA—Sabouraud dextrose agar.

Statistically significant differences among microbial counts for individual cultivation media were found in three of the four cultivation media used (Table 6).

Table 6. Significant differences among analyzed federweisser samples for individual cultivation media.

\begin{tabular}{|c|c|c|}
\hline Reatments Pair & Tukey HSD $p$-Value & Tukey HSD Inferfence \\
\hline \multicolumn{3}{|l|}{ MEA } \\
\hline A vs. $\mathrm{H}$ & 0.0368638 & ${ }^{*} p<0.05$ \\
\hline A vs. I & 0.0238533 & ${ }^{*} p<0.05$ \\
\hline A vs. J & 0.0055561 & $* * p<0.01$ \\
\hline B vs. $\mathrm{H}$ & 0.0456379 & ${ }^{*} p<0.05$ \\
\hline B vs. I & 0.0296858 & ${ }^{*} p<0.05$ \\
\hline B vs. J & 0.0069685 & $* * p<0.01$ \\
\hline $\mathrm{C}$ vs. $\mathrm{H}$ & 0.0410343 & ${ }^{*} p<0.05$ \\
\hline C vs. I & 0.0266151 & ${ }^{*} p<0.05$ \\
\hline C vs. J & 0.0062207 & $* * p<0.01$ \\
\hline E vs. H & 0.0456379 & ${ }^{*} p<0.05$ \\
\hline E vs. I & 0.0296858 & ${ }^{*} p<0.05$ \\
\hline E vs. J & 0.0069685 & $* * p<0.01$ \\
\hline \multicolumn{3}{|l|}{ WA } \\
\hline C vs. H & 0.0329699 & ${ }^{*} p<0.05$ \\
\hline C vs. I & 0.0100494 & ${ }^{*} p<0.05$ \\
\hline C vs. J & 0.0010463 & $* * p<0.01$ \\
\hline D vs. J & 0.0084520 & $* * p<0.01$ \\
\hline Evs. J & 0.0071077 & $* * p<0.01$ \\
\hline \multicolumn{3}{|l|}{ YPDA } \\
\hline D vs. J & 0.0105986 & ${ }^{*} p<0.05$ \\
\hline Evs. J & 0.0359336 & ${ }^{*} p<0.05$ \\
\hline \multicolumn{3}{|l|}{ SDA } \\
\hline B vs. J & 0.0319805 & ${ }^{*} p<0.05$ \\
\hline C vs. I & 0.0217427 & $* p<0.05$ \\
\hline C vs. J & 0.0044497 & $* * p<0.01$ \\
\hline
\end{tabular}


Table 6. Cont.

\begin{tabular}{ccc}
\hline Reatments Pair & Tukey HSD $p$-Value & Tukey HSD Inferfence \\
\hline SDA & & \\
\hline D vs. I & 0.0081105 & $* * p<0.01$ \\
D vs. J & 0.0016306 & $* * p<0.01$ \\
E vs. I & 0.0036406 & $* * p<0.01$ \\
E vs. J & 0.0010053 & $* p<0.01$ \\
F vs. I & 0.0466723 & $* * 0.05$ \\
F vs. J & 0.0098975 & $* p<0.01$ \\
G vs. J & 0.0178947 &
\end{tabular}

A-Green Veltliner; B-Mūller Thurgau; C-Palava; D-Rhein Riesling; E-Sauvignon Blanc; F-Alibernet; G-André; H-Blue Frankish; I-Cabernet Sauvignon; J-Dornfelder; WA-wort agar; YPDA-yeast extract peptone dextrose agar; MEA—malt extract agar; SDA—Sabouraud dextrose agar.

The yeast counts in the unfiltered wines are summarized in Table 7 . The yeast counts in wine ranged from 1.51 (Greener Veltliner) to 3.23 (Dornfelder) $\log \mathrm{CFU} / \mathrm{mL}$ on MEA, from 1.43 (Greener Veltliner) to 2.89 (Dornfelder) $\log \mathrm{CFU} / \mathrm{mL}$ on WA, from 1.18 (Greener Veltliner) to 2.65 (Dornfelder) $\log \mathrm{CFU} / \mathrm{mL}$ on YPDA and from 1.09 (Rhein Riesling) to 2.21 (Dornfelder) log CFU/mL on SDA.

Table 7. Yeast counts in wine on different media.

\begin{tabular}{ccccc}
\hline \multirow{2}{*}{ Sample } & MEA & WA & YPDA & SDA \\
\cline { 2 - 5 } & \multicolumn{4}{c}{$\log$ CFU/g } \\
\hline Green Veltliner & $1.51 \pm 0.27$ & $1.43 \pm 0.15$ & $1.18 \pm 0.06$ & $1.13 \pm 0.03$ \\
Mūller Thurgau & $1.55 \pm 0.32$ & $1.52 \pm 0.01$ & $1.21 \pm 0.06$ & $1.18 \pm 0.06$ \\
Palava & $1.62 \pm 0.34$ & $1.49 \pm 0.03$ & $1.27 \pm 0.11$ & $1.12 \pm 0.02$ \\
Rhein Riesling & $1.73 \pm 0.17$ & $1.46 \pm 0.05$ & $1.27 \pm 0.14$ & $1.09 \pm 0.06$ \\
Sauvignon Blanc & $1.76 \pm 0.11$ & $1.48 \pm 0.06$ & $1.32 \pm 0.23$ & $1.14 \pm 0.02$ \\
Alibernet & $2.57 \pm 0.50$ & $2.37 \pm 0.14$ & $2.21 \pm 0.05$ & $1.51 \pm 0.64$ \\
André & $2.67 \pm 0.42$ & $2.41 \pm 0.10$ & $2.31 \pm 0.13$ & $1.83 \pm 0.62$ \\
Blue Frankish & $2.59 \pm 0.28$ & $2.44 \pm 0.16$ & $2.38 \pm 0.11$ & $2.13 \pm 0.12$ \\
Cabernet Sauvignon & $2.89 \pm 0.37$ & $2.51 \pm 0.14$ & $2.47 \pm 0.04$ & $2.17 \pm 0.07$ \\
Dornfelder & $3.23 \pm 0.02$ & $2.89 \pm 0.27$ & $2.65 \pm 0.22$ & $2.21 \pm 0.06$ \\
\hline
\end{tabular}

WA—wort agar; YPDA-yeast extract peptone dextrose agar; MEA—malt extract agar; SDA—Sabouraud dextrose agar.

ANOVA analysis was performed to inspect the significant differences among the microbial count for individual wine varieties when different cultivation media were used (Table 8).

Table 8. One-way ANOVA results for analyzed wine varieties—unfiltered wine.

\begin{tabular}{ccccccc}
\hline $\begin{array}{c}\text { Cultivation } \\
\text { Media }\end{array}$ & Source & $\begin{array}{c}\text { Sum of } \\
\text { Squares }\end{array}$ & $\begin{array}{c}\text { Degrees of } \\
\text { Freedom }\end{array}$ & $\begin{array}{c}\text { Mean } \\
\text { Square }\end{array}$ & F Statistic & $p$-Value \\
\hline \multirow{3}{*}{ MEA } & treatment & 11.0908 & 9 & 1.23 & 12.60 & $1.95 \times 10^{-6}$ \\
& error & 1.45 & 20 & 0.0982 & & \\
& total & 13.0552 & 29 & & & \\
\hline \multirow{3}{*}{ WA } & treatment & 8.05 & 9 & 0.9745 & 54.8807 & $3.84 \times 10^{-12}$ \\
& error & 0.3551 & 20 & 0.0178 & & \\
& total & 9.1256 & 29 & & & \\
\hline \multirow{4}{*}{ YPDA } & treatment & 10.74 & 9 & 1.1542 & 65.9142 & $6.70 \times 10^{-13}$ \\
& error & 0.3502 & 20 & 0.0175 & & \\
& total & 10.76 & 29 & & & \\
\hline \multirow{3}{*}{ SDA } & treatment & 6.61 & 9 & 0.7118 & 8.13 & $3.51 \times 10^{-5}$ \\
& error & 1.31 & 20 & 0.0827 & & \\
& total & 8.0592 & 29 & & & \\
\hline
\end{tabular}

WA-wort agar; YPDA-yeast extract peptone dextrose agar; MEA-malt extract agar; SDA-Sabouraud dextrose agar. 
Statistically significant differences among microbial count for individual cultivation media were found in three of the four cultivation media used (Table 9).

Table 9. Significant differences among unfiltered wine samples for individual cultivation media.

\begin{tabular}{|c|c|c|}
\hline Treatments Pair & Tukey HSD $p$-Value & Tukey HSD Inferfence \\
\hline \multicolumn{3}{|l|}{ MEA } \\
\hline A vs. F & 0.0141715 & ${ }^{*} p<0.05$ \\
\hline A vs. G & 0.0064264 & $* * p<0.01$ \\
\hline A vs. H & 0.0119733 & ${ }^{*} p<0.05$ \\
\hline A vs. I & 0.0010053 & $* * * 0.01$ \\
\hline A vs. J & 0.0010053 & $* * p<0.01$ \\
\hline B vs. F & 0.0192566 & ${ }^{*} p<0.05$ \\
\hline B vs. G & 0.0087792 & $* * p<0.01$ \\
\hline B vs. H & 0.0162964 & ${ }^{*} p<0.05$ \\
\hline B vs. I & 0.0012652 & $* * p<0.01$ \\
\hline B vs. J & 0.0010053 & $* * p<0.01$ \\
\hline C vs. F & 0.0342577 & ${ }^{*} p<0.05$ \\
\hline C vs. G & 0.0158481 & ${ }^{*} p<0.05$ \\
\hline $\mathrm{C}$ vs. $\mathrm{H}$ & 0.0290959 & ${ }^{*} p<0.05$ \\
\hline C vs. I & 0.0023022 & $* * p<0.01$ \\
\hline C vs. J & 0.0010053 & $* * p<0.01$ \\
\hline D vs. G & 0.0381648 & ${ }^{*} p<0.05$ \\
\hline D vs. I & 0.0057354 & $* * p<0.01$ \\
\hline D vs. J & 0.0010053 & $* * p<0.01$ \\
\hline E vs. I & 0.0078360 & $* * p<0.01$ \\
\hline E vs. J & 0.0010053 & $* * p<0.01$ \\
\hline \multicolumn{3}{|l|}{ WA } \\
\hline A vs. F & 0.0010053 & $* * p<0.01$ \\
\hline A vs. G & 0.0010053 & $* * p<0.01$ \\
\hline A vs. $\mathrm{H}$ & 0.0010053 & $* * p<0.01$ \\
\hline A vs. I & 0.0010053 & $* * p<0.01$ \\
\hline A vs. J & 0.0010053 & $* * p<0.01$ \\
\hline B vs. F & 0.0010053 & $* * p<0.01$ \\
\hline B vs. G & 0.0010053 & $* * p<0.01$ \\
\hline B vs. $\mathrm{H}$ & 0.0010053 & $* * p<0.01$ \\
\hline B vs. I & 0.0010053 & $* * p<0.01$ \\
\hline B vs. J & 0.0010053 & $* * p<0.01$ \\
\hline C vs. F & 0.0010053 & $* * p<0.01$ \\
\hline C vs. G & 0.0010053 & $* * p<0.01$ \\
\hline $\mathrm{C}$ vs. $\mathrm{H}$ & 0.0010053 & $* * p<0.01$ \\
\hline C vs. I & 0.0010053 & $* * p<0.01$ \\
\hline C vs. J & 0.0010053 & $* * p<0.01$ \\
\hline D vs. F & 0.0010053 & $* * p<0.01$ \\
\hline D vs. G & 0.0010053 & $* * p<0.01$ \\
\hline D vs. H & 0.0010053 & $* * p<0.01$ \\
\hline D vs. I & 0.0010053 & $* * p<0.01$ \\
\hline D vs. J & 0.0010053 & $* * p<0.01$ \\
\hline E vs. F & 0.0010053 & $* * p<0.01$ \\
\hline E vs. G & 0.0010053 & $* * p<0.01$ \\
\hline E vs. H & 0.0010053 & $* * p<0.01$ \\
\hline E vs. I & 0.0010053 & $* * p<0.01$ \\
\hline E vs. J & 0.0010053 & $* * p<0.01$ \\
\hline F vs. J & 0.0037886 & $* * p<0.01$ \\
\hline G vs. J & 0.0079079 & $* * p<0.01$ \\
\hline H vs. J & 0.0143649 & ${ }^{*} p<0.05$ \\
\hline
\end{tabular}


Table 9. Cont.

\begin{tabular}{|c|c|c|}
\hline Treatments Pair & Tukey HSD $p$-Value & Tukey HSD Inferfence \\
\hline \multicolumn{3}{|l|}{ YPDA } \\
\hline A vs. F & 0.0010053 & $* * p<0.01$ \\
\hline A vs. G & 0.0010053 & $* * p<0.01$ \\
\hline A vs. H & 0.0010053 & $* * p<0.01$ \\
\hline A vs. I & 0.0010053 & $* * p<0.01$ \\
\hline A vs. J & 0.0010053 & $* * p<0.01$ \\
\hline B vs. F & 0.0010053 & $* * p<0.01$ \\
\hline B vs. $G$ & 0.0010053 & $* * p<0.01$ \\
\hline B vs. $\mathrm{H}$ & 0.0010053 & $* * p<0.01$ \\
\hline B vs. I & 0.0010053 & $* * p<0.01$ \\
\hline B vs. J & 0.0010053 & $* * p<0.01$ \\
\hline C vs. F & 0.0010053 & $* * p<0.01$ \\
\hline C vs. G & 0.0010053 & $* * p<0.01$ \\
\hline C vs. H & 0.0010053 & $* * p<0.01$ \\
\hline C vs. I & 0.0010053 & $* * p<0.01$ \\
\hline C vs. J & 0.0010053 & $* * p<0.01$ \\
\hline D vs. F & 0.0010053 & $* * p<0.01$ \\
\hline D vs. G & 0.0010053 & $* * p<0.01$ \\
\hline D vs. H & 0.0010053 & $* * p<0.01$ \\
\hline D vs. I & 0.0010053 & $* * p<0.01$ \\
\hline D vs. J & 0.0010053 & $* * p<0.01$ \\
\hline Evs. F & 0.0010053 & $* * p<0.01$ \\
\hline E vs. G & 0.0010053 & $* * p<0.01$ \\
\hline E vs. H & 0.0010053 & $* * p<0.01$ \\
\hline E vs. I & 0.0010053 & $* * p<0.01$ \\
\hline E vs. J & 0.0010053 & $* * p<0.01$ \\
\hline F vs. J & 0.0154088 & ${ }^{*} p<0.05$ \\
\hline \multicolumn{3}{|l|}{ SDA } \\
\hline A vs. $H$ & 0.0103393 & $* p<0.05$ \\
\hline A vs. I & 0.0071377 & $* * \quad p<0.01$ \\
\hline A vs. J & 0.0044827 & $* * p<0.01$ \\
\hline B vs. H & 0.0168657 & ${ }^{*} p<0.05$ \\
\hline B vs. I & 0.0116918 & ${ }^{*} p<0.05$ \\
\hline B vs. J & 0.0073639 & $* * p<0.01$ \\
\hline $\mathrm{C}$ vs. $\mathrm{H}$ & 0.0100244 & ${ }^{*} p<0.05$ \\
\hline C vs. I & 0.0069202 & $* * p<0.01$ \\
\hline C vs. J & 0.0043452 & $* * p<0.01$ \\
\hline D vs. H & 0.0075929 & $* * p<0.01$ \\
\hline D vs. I & 0.0052344 & $* * p<0.01$ \\
\hline D vs. J & 0.0032871 & $* * p<0.01$ \\
\hline E vs. H & 0.0116918 & $* p<0.05$ \\
\hline E vs. I & 0.0080777 & $* * p<0.01$ \\
\hline E vs. J & 0.0050746 & $* * p<0.01$ \\
\hline
\end{tabular}

A-Green Veltliner; B-Mūller Thurgau; C-Palava; D-Rhein Riesling; E-Sauvignon Blanc; F-Alibernet; G-André; H-Blue Frankish; I-Cabernet Sauvignon; J-Dornfelder; WA—wort agar; YPDA—yeast extract peptone dextrose agar; MEA—-malt extract agar; SDA—Sabouraud dextrose agar.

Altogether, 1668 isolates were identified with mass spectrometry with a score of $\geq 2.0$ (Table 10). The most isolated species from grape was Hanseniaspora uvarum (70 isolates), and from "federweisser" and wine S. cerevisiae (85 and 120 isolates, respectively). Yeasts species of grape, "frederweisser" and wine are shown in Figures 1-3. 
Table 10. Yeasts species in grape, "federweisser" and wine.

\begin{tabular}{|c|c|c|c|}
\hline \multirow{2}{*}{ Yeast Species } & \multirow{2}{*}{ Grape } & "Federweisser" & \multirow{2}{*}{ Wine } \\
\hline & & No. of Isolates & \\
\hline Aureobasidium pullulans & 25 & 0 & 0 \\
\hline Candida inconspicua & 5 & 0 & 5 \\
\hline Candida parapsilosis & 5 & 0 & 10 \\
\hline Candida saitoana & 5 & 0 & 5 \\
\hline Candida sake & 5 & 0 & 5 \\
\hline Cyberlindnera jadinii & 0 & 0 & 8 \\
\hline Debaryomyces hansenii & 0 & 0 & 15 \\
\hline Dekkera bruxellensis & 0 & 0 & 25 \\
\hline Filobasidium magnum & 30 & 0 & 0 \\
\hline Hanseniaspora uvarum & 70 & 25 & 0 \\
\hline Issatchenkia orientalis & 38 & 0 & 0 \\
\hline Kazachstania exigua & 33 & 0 & 0 \\
\hline Kluyveromyces marxianus & 35 & 0 & 32 \\
\hline Kregervanrija fluxuum & 0 & 0 & 25 \\
\hline Metschnikowia pulcherrima & 28 & 50 & 55 \\
\hline Meyerozyma guilliermondii & 0 & 0 & 52 \\
\hline Naganishia diffluens & 25 & 0 & 0 \\
\hline Pichia fermentans & 10 & 0 & 58 \\
\hline Pichia kluyveri & 12 & 49 & 50 \\
\hline Pichia mandshurica & 10 & 0 & 25 \\
\hline Pichia membranifaciens & 25 & 0 & 15 \\
\hline Pichia norvegensis & 10 & 0 & 5 \\
\hline Pichia occidentalis & 10 & 35 & 45 \\
\hline Rhodotorula glutinis & 40 & 0 & 20 \\
\hline Rhodotorula mucilaginosa & 25 & 0 & 45 \\
\hline Saccharomyces cerevisiae & 0 & 85 & 120 \\
\hline Starmerella magnolia & 30 & 0 & 15 \\
\hline Torulaspora delbrueckii & 12 & 0 & 39 \\
\hline Wickerhamomyces anomalus & 15 & 0 & 75 \\
\hline Yarrowia lipolytica & 20 & 0 & 10 \\
\hline Zygosaccharomyces bailii & 15 & 0 & 52 \\
\hline Zygotorulaspora florentina & 25 & 0 & 50 \\
\hline Total & 563 & 244 & 861 \\
\hline
\end{tabular}

Brettanomyces bruxellensis, Candida stellata, Saccharomyces cerevisiae and Zygosaccharomyces bailii were the yeasts identified in wine [22-25]. In our study, Pichia mandshurica-the main contaminant of wines-was present in $66 \%$ samples of white wines (10 out of 15) and in seven samples of red wines $(46 \%)$. Pichia membranifaciens was isolated from five samples of white (33\%) and five samples of red wines (33\%). Saccharomyces cerevisiae was isolated from all white and red wines $(100 \%)$. Zygosaccharomyces bailii was found in 14 samples of white (93\%) and two samples of red (13\%) wines. Our study shows that Z. bailii and P. mandshurica were isolated more frequently from white than from red wines, while $S$. cerevisiae was identified in white and red wines. The occurrence of Pichia manshurica and S. cerevisiae was different between the wine samples. According to Thomas [26], the presence of Zygosaccharomyces in wine is unacceptable in terms of wine quality. The author has stated that the minimum number of yeast present in wine spoils the product under appropriate conditions [26]. Saccharomyces cerevisiae, Debaryomyces hansenii, Wickerhamomyces anomalus (Pichia anomala), Pichia membranifaciens, Rhodotorula glutinis, Rhodotorula mucilaginosa, Torulaspora delbrueckii, Kluyveromyces marxianus, Issatchenkia orientalis, Zygosaccharomyces bailii parapsilosis, Pichia fermentans and Hanseniaspora uvarum are frequent contaminants of wines as well [27,28]. However, Renous [29] did not describe associations between wine and Pichia manshurica, Kregervanrija fluxuum (Candida vini), Candida inconspicua and Zygotorulaspora florentina. Saez [30] found that S. cerevisiae (13.93\%), 
Wickerhamomyces anomalus (8.72\%), Pichia fermentans (6.74\%) and Metschnikowia pulcherrima (6.39\%) were the most abundant in wine. Pichia (Pichia manshurica, P. membranifaciens) and Brettanomyces are producing volatile phenols, thereby affecting the quality of the wine [30].

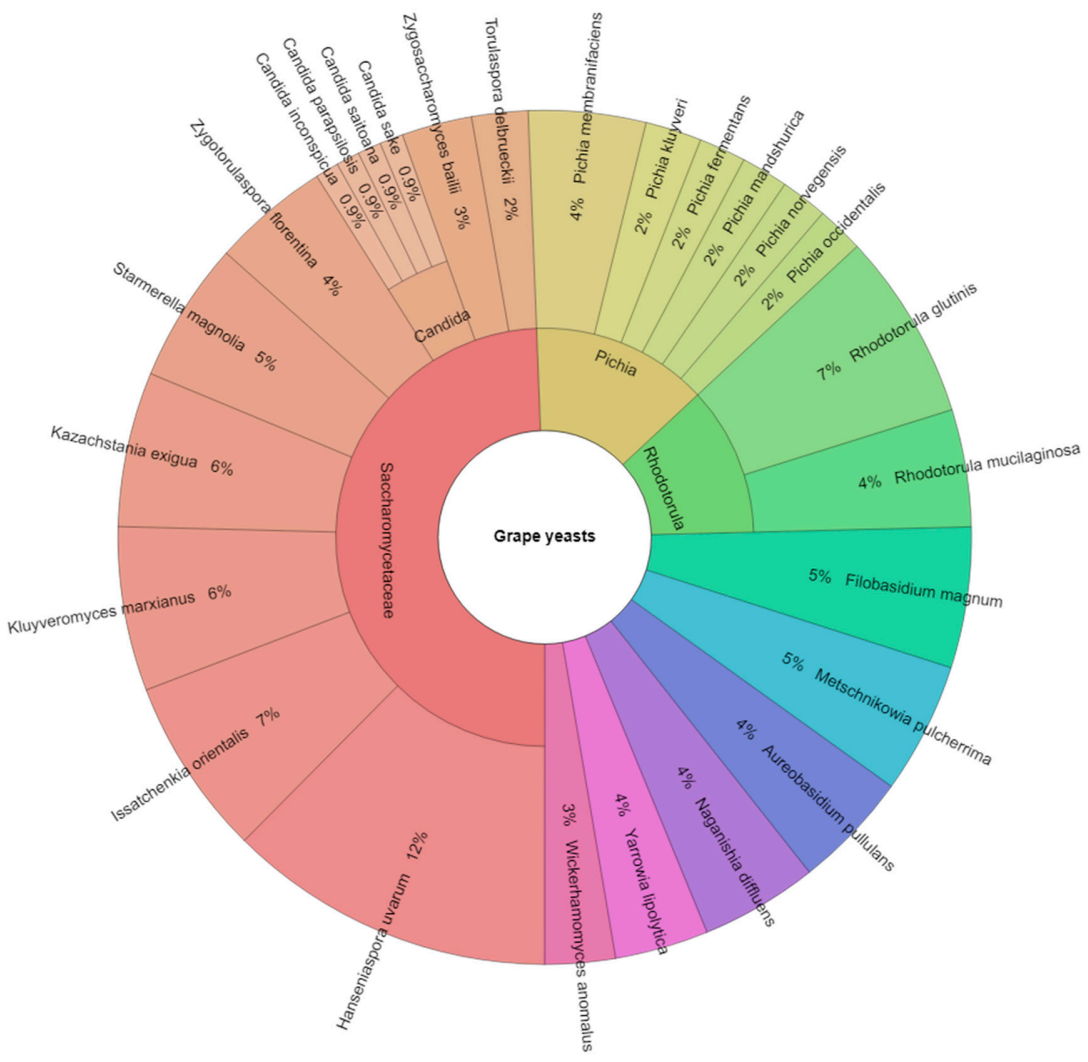

Figure 1. Yeasts isolated from the grapes.

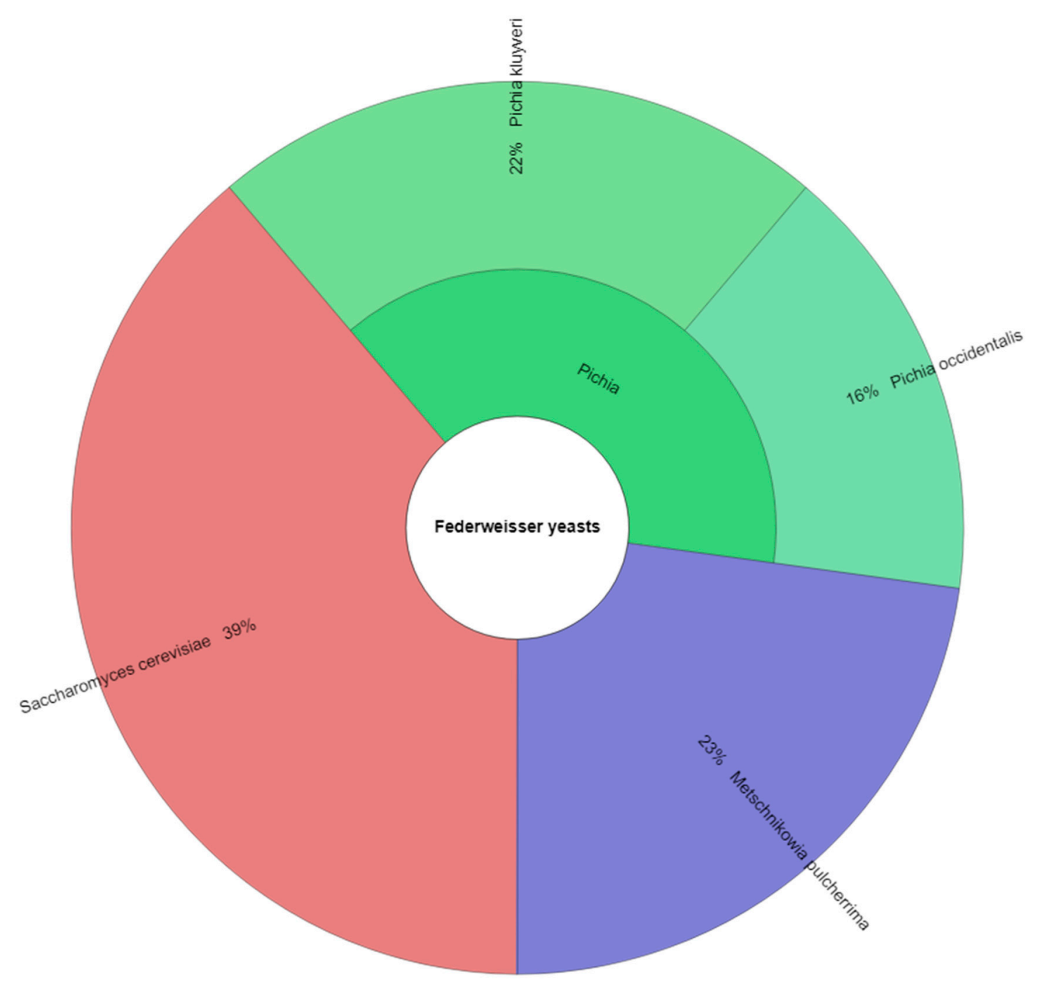

Figure 2. Yeasts isolated from the "federweisser". 


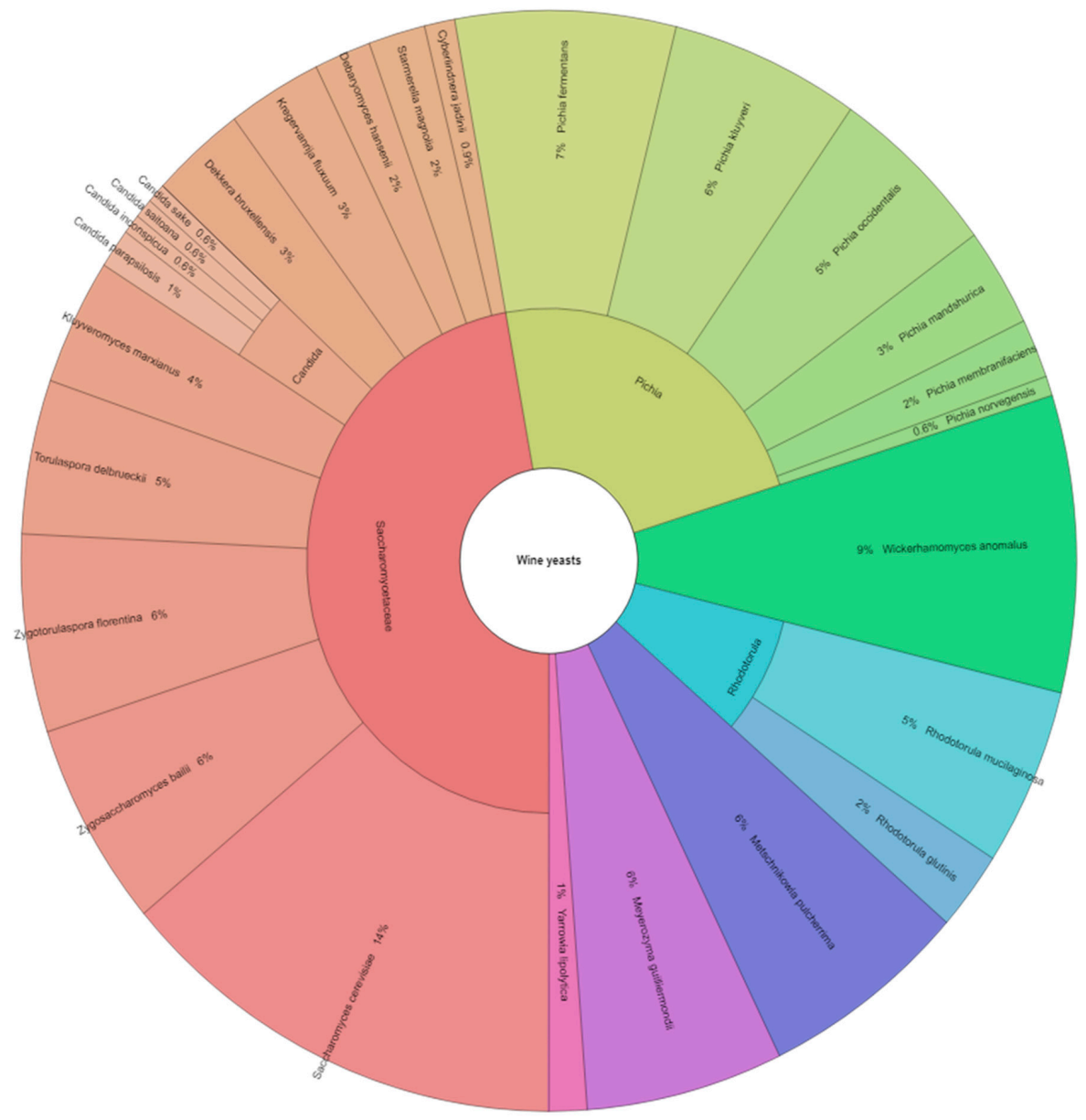

Figure 3. Yeasts isolated from the wine.

Sporadically, Candida inconspicua (5 isolates, $0.58 \%$ ), Candida saitoana ( 5 isolates, $0.58 \%$ ), Candida sake (5 isolates, $0.58 \%$ ), Pichia norvegensis ( 5 isolates, $0.58 \%$ ) and other species were isolated. Jolly et al. [31] noticed the importance of Candida, Cryptococcus, Kloeckera and Rhodotorula species in the wine making process. Candida was considered as the dominant genus, including their teleomorphic stages-Candida pulcherrima (Metschnikowia pulcherrima), Candida vini (Kregervanrija fluxuum) and Candida valida (Pichia membranifaciens) [31].

\section{Conclusions}

A total of 90 samples (30 from grapes, 30 of "federweisser" and 30 of wine) was studied for characterization of the yeast species. The mass spectrometry method was used for identification of 1668 grape, "federweisser" and wine isolates. From grape, 26 species of 17 genera within 9 families, and in "federweisser" 4 species of 3 genera and families were found. In wine, 26 species of 17 genera within 6 families were identified. Rhodoturulla species were not included in any family and they were classified as incertae sedis (not belonging anywhere).

Author Contributions: M.K., M.T., J.Ž. were responsible for the design of the study; M.K., S.K., J.S., S.F., conducted the study and collected the samples; M.K., S.K., J.S., J.Ž. performed the laboratory analysis; M.K., S.K., E.I., M.T. were responsible for writing and editing the manuscript; all authors have carefully revised and approved the final version of the manuscript. All authors have read and agreed to the published version of the manuscript.

Funding: This work has been supported by the grants of the Slovak Research and Development Agency No. VEGA 1/0411/17. 
Acknowledgments: The Paper was supported by the project: The research leading to these results has received funding from the European Community under project no. 26220220180: Building Research Centre "AgroBioTech".

Conflicts of Interest: The authors declare no conflict of interest.

\section{References}

1. Padilla, B.; Gil, J.V.; Manzanares, P. Past and future of non-saccharomyces yeasts: From spoilage microorganisms to biotechnological tools for improving wine aroma complexity. Front. Microbiol. 2016, 7, 411. [CrossRef]

2. Kantor, A.; Kačániová, M.; Kluz, M. Natural microflora of wine grape berries. J. Microbiol. Biotech. Food Sci. 2015, 4, 32-36. [CrossRef]

3. Bozoudi, D.; Tsaltas, D. The multiple and versatile roles of Aureobasidium pullulans in the vitivinicultural sector. Fermentation 2018, 4, 85. [CrossRef]

4. Fugelsang, K.C.; Edwards, C.G. Wine Microbiology: Practical Applications and Procedures, 2nd ed.; Springer: New York, NY, USA, 2007; p. 393.

5. König, H.; Fröhlich, J.; Unden, G. Biology of Microorganisms on Grapes, in Must and in Wine; Springer: Berlin/Heidelberg, Germany, 2009; p. 522.

6. Fleet, G.H. Wine. In Food Microbiology Fundamentals E Frontiers; Doyle, M.P., Beuchat, L.R., Montville, T.J., Eds.; ASM Press: Washington, DC, USA, 2001; pp. 747-772.

7. Romano, P.; Fiore, C.; Paraggio, M.; Caruso, M.; Capece, A. Function of yeast species and strains in wine flavor. Int. J. Food Microbiol. 2003, 86, 169-180. [CrossRef]

8. Kurtzman, C.P.; Fell, J.W.; Boekhout, T. The Yeasts: A Taxonomic Study, 5th ed.; Elsevier Science: Amsterdam, The Netherlands, 2011; p. 2384.

9. Kačániová, M.; Mellen, M.; Vukovic, N.L.; Kluz, M.; Puchalski, C.; Haščík, P.; Kunová, S. Combined effect of vacuum packaging, fennel and svory essential oil treatment on the quality of chicken thighs. Microorganisms 2019, 7, 134. [CrossRef] [PubMed]

10. Barata, A.; Malfeito-Ferreira, M.; Loureiro, V. The microbial ecology of wine grape berries. Int. J. Food Microbiol. 2012, 153, 243-259. [CrossRef]

11. Martins, G.; Miot-Sertier, C.; Lauga, B.; Claisse, O.; Lonvaud-Funel, A.; Soulas, G.; Masneuf-Pomarède, I. Grape berry bacterial microbiota: Impact of the ripening process and the farming system. Int. J. Food Microbiol. 2012, 158, 93-100. [CrossRef]

12. Martins, G.; Vallance, J.; Mercier, A.; Albertin, W.; Stamatopoulos, P.; Rey, P.; Lonvaud, A.; MasneufPomarède, I. Influence of the farming system on the epiphytic yeasts and yeast-like fungi colonizing grape berries during the ripening process. Int. J. Food Microbiol. 2014, 177, 21-28. [CrossRef]

13. Setati, M.E.; Jacobson, D.; Andong, U.C.; Bauer, F. The vineyard yeast microbiome, a mixed model microbial map. PLoS ONE 2012, 7, e52609. [CrossRef]

14. Bokulich, N.A.; Thorngate, J.H.; Richardson, P.M.; Mills, D.A. Microbial biogeography of wine grapes is conditioned by cultivar, vintage, and climate. Proc. Natl. Acad. Sci. USA 2014, 111, E139-E148. [CrossRef]

15. Portillo, M.; Del, C.; Franquès, J.; Araque, I.; Reguant, C.; Bordons, A. Bacterial diversity of Grenache and Carignan grape surface from different vineyards at Priorat wine region (Catalonia, Spain). Int. J. Food Microbiol. 2016, 219, 56-63. [CrossRef] [PubMed]

16. Salvetti, E.; Campanaro, S.; Campedelli, I.; Fracchetti, F.; Gobbi, A.; Tornielli, G.B.; Torriani, S.; Felis, G.E. Whole-metagenome-sequencing-based community profiles of Vitis vinifera L. cv. Corvina berries withered in two post-harvest conditions. Front. Microbiol. 2016, 7, 937. [CrossRef]

17. Grangeteau, C.; Roullier-Gall, C.; Rousseaux, S.; Gougeon, R.D.; Schmitt-Kopplin, P.; Alexandre, H.; Guilloux-Benatier, M. Wine microbiology is driven by vineyard and winery anthropogenic factors. Microb. Biotechnol. 2017, 10, 354-370. [CrossRef] [PubMed]

18. Oliveira, M.; Arenas, M.; Lage, O.; Cunha, M.; Amorim, M.I. Epiphytic fungal community in Vitis vinifera of the Portuguese wine regions. Lett. Appl. Microbiol. 2018, 66, 93-102. [CrossRef] [PubMed]

19. Kántor, A.; Petrová, J.; Hutková, J.; Kačániová, M. Yeast diversity in new, still fermenting wine "federweisser". Potravinarstvo 2016, 10, 120-125. [CrossRef]

20. Malík, F.; Furdíková, K.; Roman, T.; Malík, F. Vinársky Lexicon, 1st ed.; Fedor Malík a syn: Modra, Slovakia, 2012; p. 144. 
21. Ribéreau-Gayon, P.; Dubourdieu, D.; Donèche, B.; Lonvaud, A. Handbook of Enology, the Microbiology of Wine and Vinifications; John Wiley \& Sons Ltd.: West Sussex, UK, 2006; Volume 1, p. 512.

22. Millet, V.; Lonvaud-Funel, A. The viable but non-culturable state of wine microorganisms during storage. Lett. Appl. Microbiol. 2000, 30, 136-141. [CrossRef]

23. Divol, B.; Lonvaud-Funel, A. Evidence for viable but non-culturable yeasts in botrytis-affected wine. J. Appl. Microbiol. 2005, 99, 85-93. [CrossRef]

24. Du Toit, W.J.; Pretorius, I.S.; Lonvaud-Funel, A. The effect of suphur dioxide and oxygen on the viability and culturability of a strain of Acetobacter pasteurianus and a strain of Brettanomyces bruxellensis isolated from wine. J. Appl. Microbiol. 2005, 98, 862-871. [CrossRef]

25. Oliver, J.D. The viable but non-culturable state in bacteria. J. Microbiol. 2005, 43, 93-100.

26. Thomas, D.S. Yeasts as spoilage organisms in beverages. In The Yeasts, 2nd ed.; Rose, A.H., Harrison, J.S., Eds.; Academic Press: London, UK, 1993; Volume 5, pp. 517-561.

27. Deák, T.; Beuchat, L.R. Yeasts associated with fruit juice concentrates. J. Food Protect. 1993, 56, 777-782. [CrossRef]

28. Loureiro, V.; Malfeito-Ferreira, M. Spoilage yeasts in the wine industry (review). Int. J. Food Microbiol. 2003, 86, 23-50. [CrossRef]

29. Renouf, V.; Lonvaud-Funel, A. Development of an enrichment medium to detect Dekkera/Brettanomyces bruxellensis, a spoilage wine yeast, on the surface of grape berries. Microbiol. Res. 2007, 162, 154-167. [CrossRef] [PubMed]

30. Saez, J.S.; Lopes, C.H.A.; Kirs, V.E.; Sangorrín, M. Production of volatile phenols by Pichia manshurica and Pichia membranifaciens isolated from spoiled wines and cellar environment in Patagonia. Food Microbiol. 2011, 28, 503-509. [CrossRef] [PubMed]

31. Jolly, N.P.; Augustyn, O.P.H.; Pretorius, I.S. The occurrence of non-Saccharomyces yeast strains over three vintages in four vineyards and grape musts 130 from four production regions of the Western Cape, South Africa. S. Afr. J. Enol. Vitic. 2003, 24, 35-42.

(C) 2020 by the authors. Licensee MDPI, Basel, Switzerland. This article is an open access article distributed under the terms and conditions of the Creative Commons Attribution (CC BY) license (http://creativecommons.org/licenses/by/4.0/). 